\title{
First-Order Solutions for the Buckling Loads of Euler-Bernoulli Weakened Columns
}

\author{
J. A. Loya ${ }^{1}$; G. Vadillo²; and J. Fernández-Sáez ${ }^{3}$
}

\begin{abstract}
In this work, closed-form expressions for the buckling loads of a weakened column with different boundary conditions are presented. The cracked-column model is based on the well-known method consisting of dividing the column into two segments connected by a rotational linear spring whose flexibility is related to the crack size and the geometry of the cross section. For the formulation of closed-form expressions, the perturbation method is used and the results are compared with those found by directly solving the eigenvalue problem.
\end{abstract}

DOI: 10.1061/(ASCE)EM.1943-7889.0000103

CE Database subject headings: Cracking; Columns; Buckling; Eigenvalues.

Author keywords: Euler-Bernoulli cracked columns; Buckling loads; Perturbation method.

\section{Introduction}

The analysis of the stability of Euler-Bernoulli columns is a fundamental topic to design structures in civil, mechanical, aeronautical, nuclear, and offshore fields. Buckling is one of the most usual modes of instability of columnlike structures. Buckling of intact columns under various end conditions was discussed by Timoshenko and Gere (1965). On the other hand, defects such as cracks can appear in structures as a consequence of manufacturing process as well during service loads. It is well-known that the presence of cracks diminishes the stiffness of a structure, leading to higher displacements for the same loads and a decrease in natural frequencies.

A widely used method to analyze the mechanical behavior of damaged (weakened) beams is to consider it as two beams connected at the cracked section by a rotational spring whose stiffness is related to the crack size and the geometry of the cross section (Freund and Herrmann 1976). This model requires the continuity of displacements, bending moment, and shear force and it promotes a discontinuity in the slope of the beam deflection proportional to the bending moment transmitted. This cracked beam model can describe not only the weakness due to a notch or

\footnotetext{
${ }^{1}$ Associate Professor, Dept. of Continuum Mechanics and Structural Analysis, Univ. Carlos III of Madrid, Avda. de la Universidad, 30, 28911 Leganés, Madrid, Spain (corresponding author). E-mail: jloya@ ing.uc $3 \mathrm{~m}$.es

${ }^{2}$ Assistant Professor, Dept. of Continuum Mechanics and Structural Analysis, Univ. Carlos III of Madrid, Avda. de la Universidad, 30, 28911 Leganés, Madrid, Spain. E-mail: gvadillo@ing.uc3m.es

${ }^{3}$ Full Professor, Dept. of Continuum Mechanics and Structural Analysis, Univ. Carlos III of Madrid, Avda. de la Universidad, 30, 28911 Leganés, Madrid, Spain. E-mail: ppfer@ing.uc3m.es
}

crack, but also the behavior of robotic arm joint. Using this concept of rotationally restrained junction, Wang et al. (2004) determined the exact buckling-load values of weakened columns with several end conditions.

Caddemi and Caliò (2008) have solved the problem of buckling of the multicracked Euler-Bernoulli column. The presence of a concentrated crack is modeled considering singularities in the flexural stiffness by means of Dirac's delta distributions. Biondi and Caddemi $(2005,2007)$ showed that this method is equivalent to the internal rotational spring.

Explicit solutions are desirable for the practice of engineering and, in this work, closed-form expressions are presented for the buckling loads of Euler-Bernoulli weakened columns with different boundary conditions. The crack is considered as an internal rotational linear spring connecting the uncracked parts of the column, whose flexibility is related to the crack size and the geometry of the cross section. The analytical expressions are derived using the perturbation method to solve the eigenvalue problem resulting from the mathematical description of a cracked column's response behavior. The results are compared with those found by applying other procedures, and validity limits are established.

\section{Buckling Loads by the Direct Method}

Let us consider a column of length $L$ and constant width $W$, cross section area $A$, and moment of inertia $I$. We consider now that the column has a crack, always open, of depth $a$, located at a distance $b$, measured from one from the supports (see Fig. 1). Following the method proposed by Freund and Herrmann 1976 and further followed by many others (Adams et al. 1978; Morassi 1993; Narkis 1994; Fernández-Sáez et al. 1999; Fernández-Sáez and Navarro 2002; Krawczuk et al. 2003; Wang et al. 2004; Loya et al. 2006), we can model the cracked beam as two beams connected at the cracked section by a rotational spring whose stiffness is related to the crack size and the geometry of the cross section. 

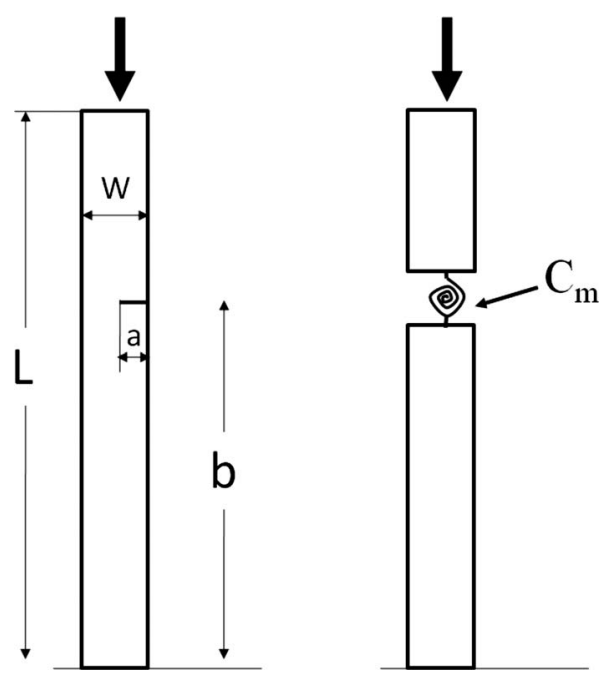

Fig. 1. Model of the cracked column

The transverse displacement of each segment of the beam, $v_{k}(x)$, obeys the following equation:

$$
E I \frac{d^{4} v_{k}(x)}{d x^{4}}+F_{c} \frac{d^{2} v_{k}(x)}{d x^{2}}=0 \quad(k=1,2)
$$

Subscript $k=1$ means upper part of the cracked column and subscript $k=2$ means lower part. This model preserves the continuity of displacement, bending moment, and shear force, and it promotes a discontinuity in the slope of the column deflection at the cracked section, $\Delta \theta$, which is proportional to the bending moment transmitted, $M(b)$

$$
\Delta \theta=C_{m} M(b)
$$

where $C_{m}=$ flexibility constant that can be expressed as

$$
C_{m}=\frac{W}{E I} m(\chi, \text { geometry of the cross section of the beam })
$$

$m$ being a function that depends on the crack depth ratio $\chi=a / W$ as well as on the geometry of the cross section of the column, and can be evaluated using the theory of fracture mechanics. For the particular case of a beam of rectangular cross section, the function $m$ takes the form (Tada et al. 1985)

$$
m(\chi)=2\left(\frac{\chi}{1-\chi}\right)^{2}\left[5.93-19.69 \chi+34.14 \chi^{2}-35.84 \chi^{3}+13.2 \chi^{4}\right]
$$

Using the new dimensionless variables and constants given by

$$
V=\frac{v}{L} \quad \xi=\frac{v}{L} \lambda^{2}=\frac{F_{c} L^{2}}{E I}
$$

we can formulate the following eigenvalue problem:

$$
V_{k}^{I V}+\lambda^{2} V_{k}^{\prime \prime}=0
$$

Eq. (6) must be solved with the corresponding end conditions and the following compatibility conditions at the cracked section $\beta=b / L$.

- Continuity of the transverse deflection

$$
V_{1}(\beta)=V_{2}(\beta)
$$

- Jump in the slope of deflection

$$
\Delta \Theta=V_{2}^{\prime}(\beta)-V_{1}^{\prime}(\beta)=\eta V_{2}^{\prime \prime}(\beta)
$$

- Continuity of the bending moment, $M$

$$
V_{2}^{\prime \prime}(\beta)=V_{1}^{\prime \prime}(\beta)
$$

- Continuity of the shear force, $Q$

$$
V_{2}^{\prime \prime \prime}(\beta)+\lambda^{2} V_{2}^{\prime}(\beta)=V_{1}^{\prime \prime \prime}(\beta)+\lambda^{2} V_{1}^{\prime}(\beta)
$$

here, $\eta=(W / L) m(\chi)$ is the crack-severity parameter.

The general solution of Eq. (6) satisfying the above compatibility conditions can be expressed as

$$
\begin{gathered}
V_{1}(\xi)=V_{0}+\frac{\theta_{0}}{\lambda} \sin (\lambda \xi)+\frac{\overline{M_{0}}}{\lambda^{2}}[1-\cos (\lambda \xi)]+\frac{\overline{Q_{0}}}{\lambda^{2}}\left[\xi-\frac{\sin (\lambda \xi)}{\lambda}\right] \\
0<\xi \leqslant \beta \\
V_{2}(\xi)=V_{1}(\xi)+\eta V_{1}^{\prime \prime}(\beta) \frac{\theta_{0}}{\lambda} \sin [\lambda(\xi-\beta)] ; \beta<\xi \leqslant 1
\end{gathered}
$$

In this expression $V_{0}, \theta_{0}, \overline{M_{0}}$, and $\overline{Q_{0}}$, are, respectively, the nondimensional displacement, slope, bending moment, and shear force at $\xi=0$. The nondimensional bending moment, $\bar{M}$, and shear force $\bar{Q}$ can be written from the true corresponding variables $M$ and $Q$ as

$$
\bar{M}=\frac{M L}{E I} ; \quad \bar{Q}=\frac{Q L^{2}}{E I}
$$

Then, imposing the end conditions, a homogeneous algebraic linear system arises. To allow a nontrivial solution, the determinant of the coefficient matrix should be zero and, consequently, the corresponding characteristic equation can be obtained. For typical boundary conditions, the following characteristic equations are derived:

Pinned-pinned

$$
\sin [\lambda]-\lambda \eta \cdot \sin [\lambda(1-\beta)] \cdot \sin [\lambda \beta]=0
$$

Clamped-free

$$
\cos [\lambda]-\eta \lambda \cdot \cos [\lambda \beta] \cdot \sin [\lambda(1-\beta)]=0
$$

Clamped-pinned

$$
\lambda \cos [\lambda]-\sin [\lambda]+\lambda \eta \sin [\lambda(1-\beta)]\{\sin [\lambda \beta]-\lambda \cos [\lambda \beta]\}=0
$$

\section{Clamped-clamped}

$$
2-\frac{1}{2}\left(4+\eta \lambda^{2}\right) \cos [\lambda]-\frac{1}{2} \eta \lambda^{2} \cos [\lambda-2 \lambda \beta]+\lambda \sin [\lambda](\eta-1)=0
$$

The above equations coincide with the ones developed by Wang et al. (2004) and they must be numerically solved to obtain the critical buckling loads. Note that for the case of undamaged column $(\eta=0), \lambda=\alpha$ with $\alpha^{2}=F L^{2} / E I$, being $F$ the buckling load corresponding to the undamaged column.

\section{Perturbation Method: First-Order Solutions}

Alternatively, the perturbation method can be applied to solve Eq. (6). The perturbative solution is reached by extending the 
method originally proposed by Morassi (1993) for the vibrations of cracked Euler-Bernoulli beams, with the assumption that the solutions for the cracked and the uncracked beams are slightly different.

Accordingly, the following expansions are introduced:

$$
\begin{gathered}
V_{1}(\xi)=V(\xi)+\varepsilon Y_{1}(\xi)+\mathrm{O}\left(\varepsilon^{2}\right) \\
V_{2}(\xi)=V(\xi)+\varepsilon Y_{2}(\xi)+\mathrm{O}\left(\varepsilon^{2}\right) \\
\lambda^{2}=\alpha^{2}+\varepsilon \mu^{2}+\mathrm{O}\left(\varepsilon^{2}\right)
\end{gathered}
$$

Here, $V(\xi)$ is the transverse displacement corresponding to the undamaged column that obeys the following differential equation:

$$
V^{I V}+\alpha^{2} V^{\prime \prime}=0
$$

and $\varepsilon=$ small parameter of the same order as the flexibility of the linear spring representing the crack. $Y_{1}(\xi), Y_{2}(\xi)$, and $\mu$ are variables of the problem that have to be determined as a part of the solution.

Substituting the new variables into Eq. (6) and keeping the first-order terms, the following equations for the new variables $Y_{1}(\xi)$, and $Y_{2}(\xi)$ are reached:

$$
\begin{aligned}
& Y_{1}^{I V}(\xi)+\alpha^{2} Y_{1}^{\prime \prime}(\xi)=-\mu^{2} V^{\prime \prime}(\xi), \quad 0<\xi<\beta \\
& Y_{2}^{I V}(\xi)+\alpha^{2} Y_{1}^{\prime \prime}(\xi)=-\mu^{2} V^{\prime \prime}(\xi), \quad \beta<\xi<1
\end{aligned}
$$

To solve these equations, we must apply the corresponding boundary and compatibility conditions. The boundary conditions are:

Simply supported end

$$
Y_{i}=Y_{i}^{\prime \prime}=0
$$

Fixed end

$$
Y_{i}=Y_{i}^{\prime}=0
$$

Free end

$$
Y_{i}^{\prime \prime}=0, \quad Y_{i}^{\prime \prime \prime}+\alpha^{2} Y_{i}^{\prime}=-\mu V^{\prime}
$$

In the above equations, $i=1$ for the upper end and $i=2$ for the lower one.

The compatibility conditions at the cracked section $(\xi=\beta)$ are: continuity of the transverse deflection

$$
Y_{2}(\beta)-Y_{1}(\beta)=0
$$

Jump in the slope deflection

$$
Y_{2}^{\prime}(\beta)-Y_{1}^{\prime}(\beta)=\frac{1}{\varepsilon} \eta V^{\prime \prime}(\beta)
$$

Continuity of the bending moment

$$
Y_{2}^{\prime \prime}(\beta)-Y_{1}^{\prime \prime}(\beta)=0
$$

Continuity of the shear force

$$
Y_{2}^{\prime \prime \prime}(\beta)-Y_{1}^{\prime \prime \prime}(\beta)+\alpha^{2}\left[Y_{2}^{\prime}(\beta)-Y_{1}^{\prime}(\beta)\right]=0
$$

Multiplying Eqs. (22) and (23) by $V(\xi)$ and integrating over the whole length of the beam, the following equation can be written:

$$
\begin{aligned}
& \int_{0}^{\beta}\left[Y_{1}^{I V}(\xi)+\alpha^{2} Y_{1}^{\prime \prime}(\xi)\right] V(\xi) d \xi+\int_{\beta}^{1}\left[Y_{2}^{I V}(\xi)+\alpha^{2} Y_{2}^{\prime \prime}(\xi)\right] V(\xi) d \xi \\
& \quad=-\mu^{2} \int_{0}^{1} V^{\prime \prime}(\xi) V(\xi) d \xi
\end{aligned}
$$

After two integrations by parts, Eq. (31) becomes

$$
H_{1}+H_{2}+H_{3}+H_{4}=-\mu^{2} H_{5}
$$

where $H_{1}, H_{2}, H_{3}, H_{4}$, and $H_{5}$ have the following expressions:

$$
H_{1}=\int_{0}^{1}\left[V^{I V}(\xi)+\alpha^{2} V^{\prime \prime}(\xi)\right] f(\xi) d \xi
$$

being

$$
\begin{gathered}
f(\xi)= \begin{cases}Y_{1}(\xi), & 0<\xi<\beta \\
Y_{2}(\xi), & \beta<\xi<1\end{cases} \\
H_{2}=Y_{1}(0)\left[V^{\prime \prime \prime}(0)+\alpha^{2} V^{\prime}(0)\right]-V(0)\left[Y_{1}^{\prime \prime \prime}(0)+\alpha^{2} Y_{1}^{\prime}(0)\right] \\
+V^{\prime}(0) Y_{1}^{\prime \prime}(0)-V^{\prime \prime}(0) Y_{1}^{\prime}(0) \\
H_{3}=V(\beta)\left\{Y_{1}^{\prime \prime \prime}(\beta)-Y_{2}^{\prime \prime \prime}(\beta)+\alpha^{2}\left[Y_{1}^{\prime}(\beta)-Y_{2}^{\prime}(\beta)\right]\right\}+V^{\prime}(\beta)\left\{Y_{2}^{\prime \prime}(\beta)\right. \\
\left.-Y_{1}^{\prime \prime}(\beta)+\alpha^{2}\left[Y_{2}(\beta)-Y_{2}(\beta)\right]\right\}+V^{\prime \prime}(\beta)\left[Y_{1}^{\prime}(\beta)-Y_{2}^{\prime}(\beta)\right] \\
+V^{\prime \prime \prime}(\beta)\left[Y_{2}(\beta)-Y_{1}(\beta)\right] \\
H_{4}=V(1)\left[Y_{2}^{\prime \prime \prime}(1)+\alpha^{2} Y_{2}^{\prime}(1)\right]-Y_{2}(1)\left[V^{\prime \prime \prime}(1)+\alpha^{2} V^{\prime}(1)\right] \\
+V^{\prime \prime}(1) Y_{2}^{\prime}(1)-V^{\prime}(1) Y_{2}^{\prime \prime}(1) \\
H_{5}=\int_{0}^{1} V^{\prime \prime}(\xi) V(\xi) d \xi
\end{gathered}
$$

Since $V(\xi)$ is the solution of Eq. (21), it follows that $H_{1}=0$. Taking into account the boundary conditions and the compatibility conditions, $\mathrm{H}_{2}=\mathrm{H}_{3}=0$, and $\mathrm{H}_{4}$ is simplified as

$$
H_{4}=-\frac{1}{\varepsilon} \eta\left[V^{\prime \prime}(\beta)\right]^{2}-\mu^{2}\left[V(1) V^{\prime}(1)-V(0) V^{\prime}(0)\right]
$$

Therefore, from Eqs. (20), (32), (38), and (39), a closed-form expression for $\lambda^{2}$ can be derived as

$$
\lambda^{2}=\alpha^{2}-\eta \frac{\left[V^{\prime \prime}(\beta)\right]^{2}}{V(1) V^{\prime}(1)-V(0) V^{\prime}(0)-\int_{0}^{1} V^{\prime \prime}(\xi) V(\xi) d \xi}
$$

This method provides expressions for the eigenvalue of buckling of Euler-Bernoulli cracked columns with simple boundary conditions from the well-known eigenvalue $\left(\alpha^{2}\right)$ and buckling modes $[V(\xi)]$ of the uncracked beam.

The above general Eq. (40) can be applied to simple boundary conditions. Here, four different cases have been considered.

Pinned-pinned column: for this case, the first eigenvalue for the uncracked column is $\alpha=\pi$, and the corresponding buckling mode is given by

$$
V(\xi)=A \sin (\pi \xi)
$$

Then, Eq. (40) takes the form

$$
\lambda^{2}=\pi^{2}\left[1-2 \eta(\sin \pi \beta)^{2}\right]
$$

and the first buckling load dimensionless with that corresponding to the uncracked column, $\bar{P}_{c}=\lambda / \alpha$, is 

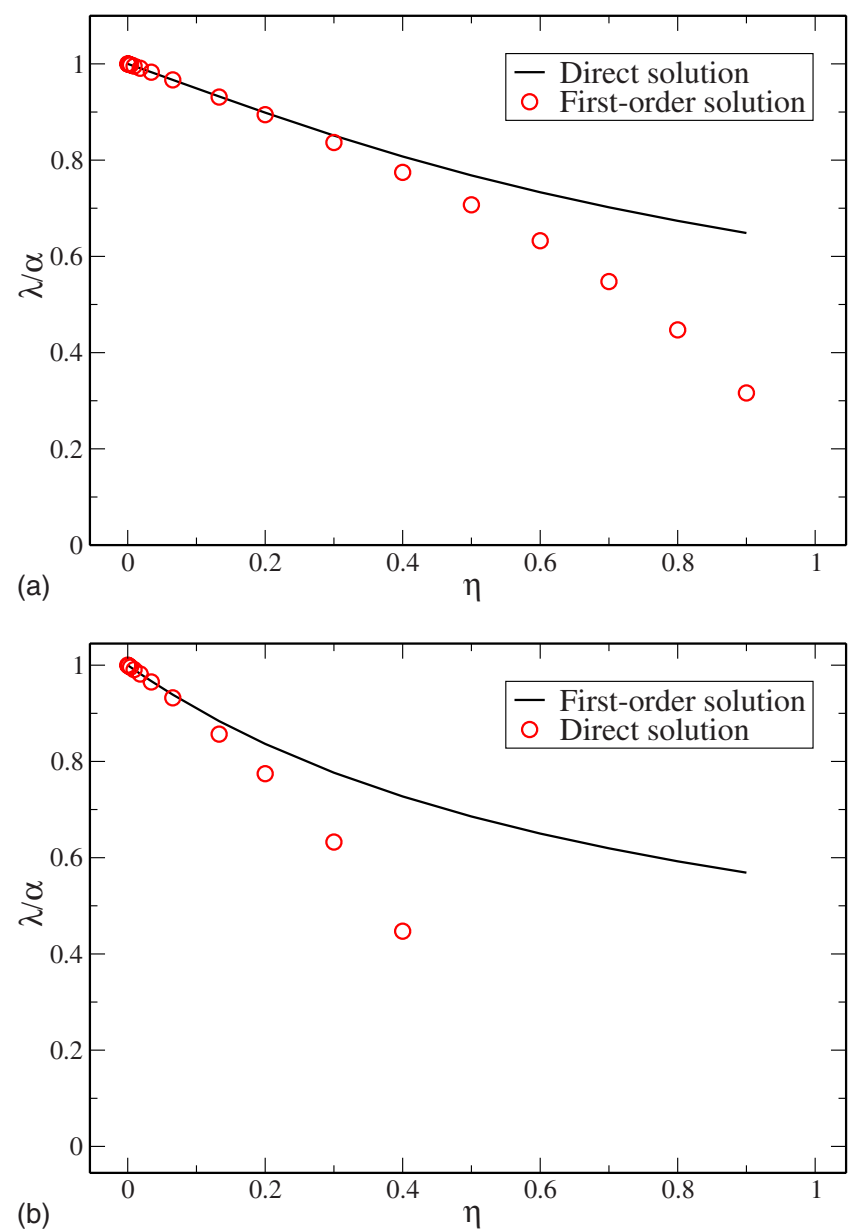

Fig. 2. First buckling load for different values of crack severity for a cracked pinned-pinned column: (a) crack located at $\beta=0.25$; (b) crack located at $\beta=0.50$

$$
\bar{P}_{c}=\sqrt{1-2 \eta \sin ^{2}(\pi \beta)}
$$

Clamped-free column

$$
\begin{gathered}
\alpha=\frac{\pi}{2} \\
V(\xi)=A\left[1-\cos \left(\frac{\pi}{2} \xi\right)\right] \\
\lambda^{2}=\frac{\pi^{2}}{4}\{1-\eta[1-\cos (\pi \beta)]\} \\
\bar{P}_{c}=\sqrt{1-\eta[1-\cos (\pi \beta)]}
\end{gathered}
$$

Clamped-pinned column

$$
\begin{gathered}
\alpha=h \pi \text { with } h=1 / \sqrt{0.699} \\
V(\xi)=A[\sin (h \pi \xi)-h \pi \xi \cos (h \pi \xi)] \\
\lambda^{2}=h^{2} \pi^{2}\left\{1-\frac{24 h \pi \eta[h \pi \beta \cos (h \pi \beta)+\sin (h \pi \beta)]^{2}}{4 \pi^{3} h^{3}-6 \pi \cos (2 h \pi) h+\left(3-6 h^{2} \pi^{2}\right) \sin (2 h \pi)}\right\}
\end{gathered}
$$
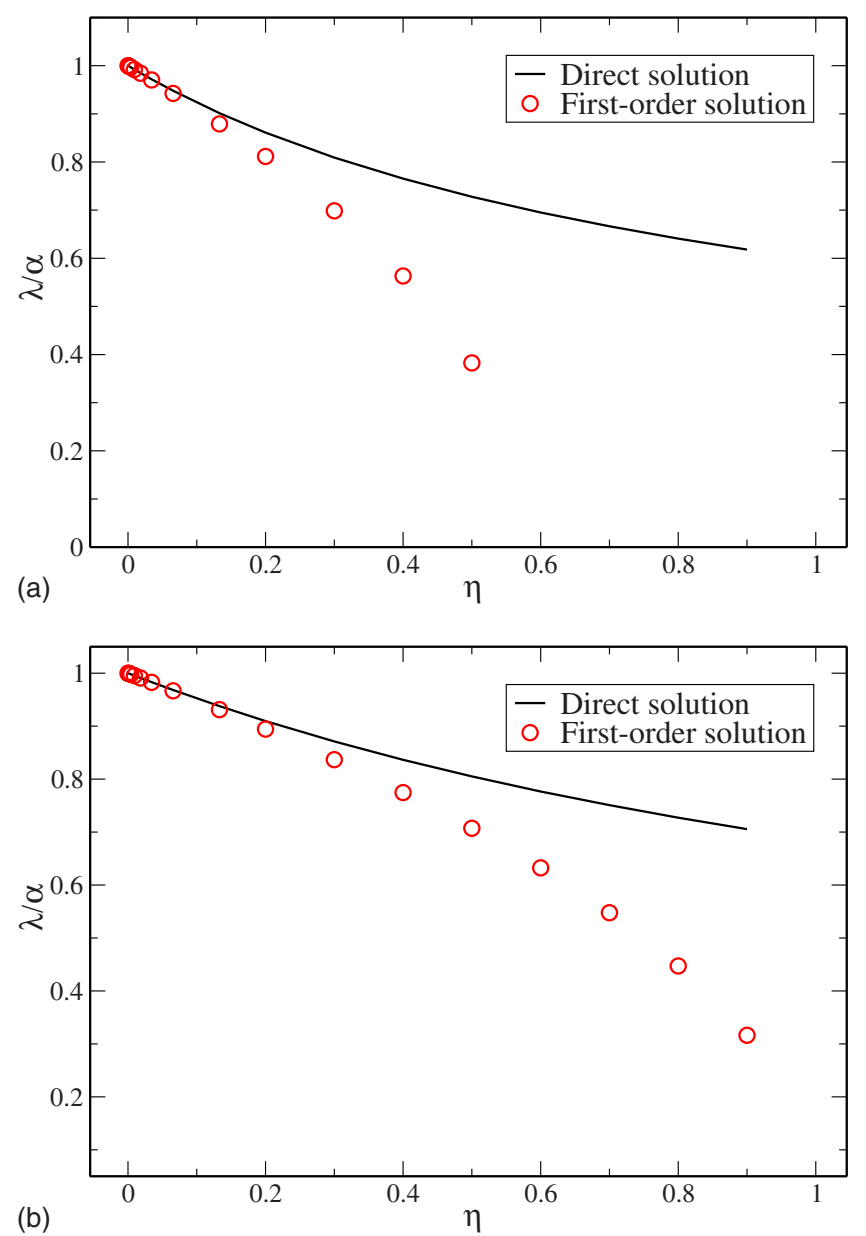

Fig. 3. First buckling load for different values of crack severity for a cracked clamped-free column: (a) crack located at $\beta=0.25$; (b) crack located at $\beta=0.50$

$$
\bar{P}_{c}=\sqrt{1-\frac{24 h \pi \eta[h \pi \beta \cos (h \pi \beta)+\sin (h \pi \beta)]^{2}}{4 \pi^{3} h^{3}-6 \pi \cos (2 h \pi) h+\left(3-6 h^{2} \pi^{2}\right) \sin (2 h \pi)}}
$$

Clamped-clamped column

$$
\begin{gathered}
\alpha=2 \pi \\
V(\xi)=A[\cos (2 \pi \xi)-1] \\
\lambda^{2}=4 \pi^{2}\left[1-2 \eta \cos ^{2}(2 \pi \beta)\right] \\
\bar{P}_{c}=\sqrt{\left[1-2 \eta \cos ^{2}(2 \pi \beta)\right]}
\end{gathered}
$$

\section{Results}

To check the accuracy of the proposed method, a comparison between the first buckling load determined by the direct method ("Buckling Loads by the Direct Method" section) and by the perturbation method ("Perturbation Method. First-Order Solutions" section) was performed for several cases corresponding to the different boundary conditions. Fig. 2 shows the variation of the first buckling load with crack severity $\eta$, of a simply sup- 

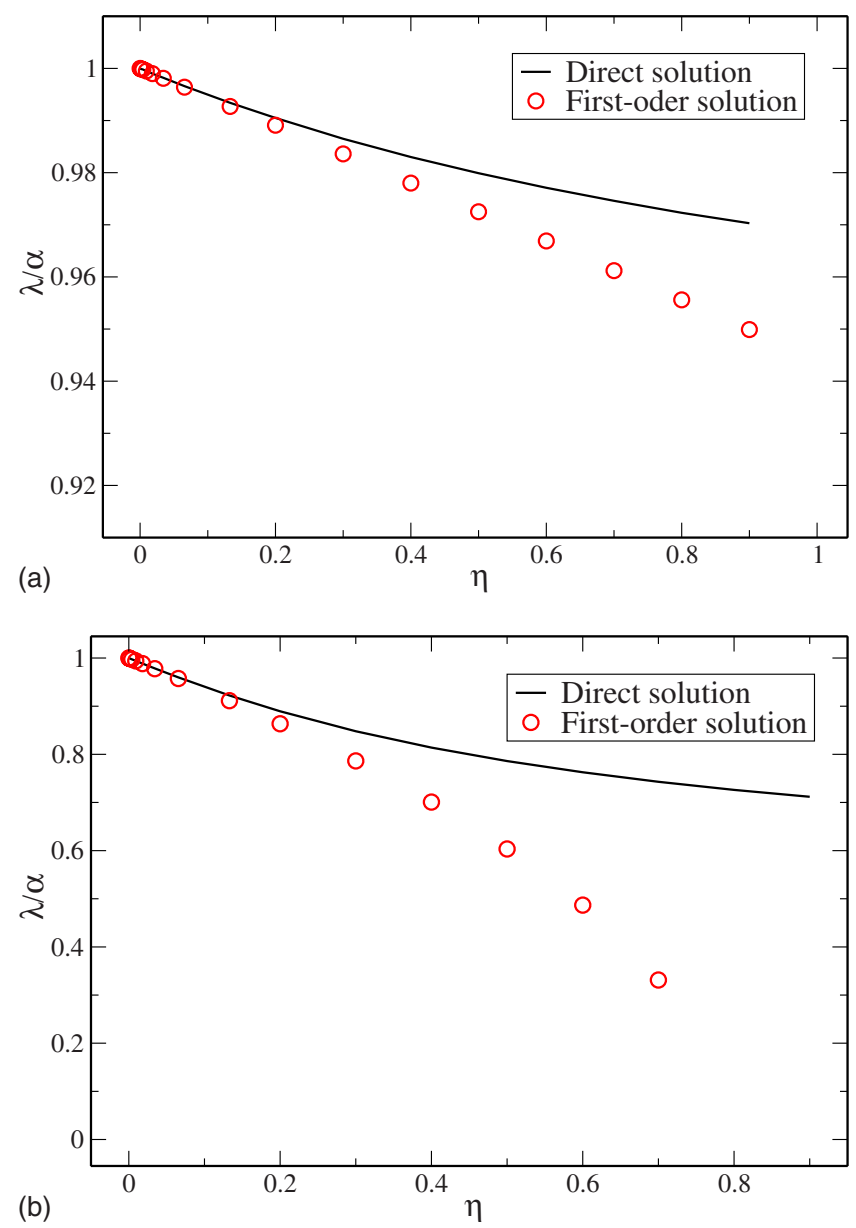

Fig. 4. First buckling load for different values of crack severity for a cracked clamped-pinned column: (a) crack located at $\beta=0.25$; (b) crack located at $\beta=0.50$

ported column containing a crack located at section $\beta=0.25$ [Fig. 2(a)] and $\beta=0.50$ [Fig. 2(b)]. The first-order solution differs from the direct solution less than 5\% until a $\eta$ value of 0.16 for a crack located at $\beta=0.25$, and $\eta=0.46$ for a crack located at $\beta=0.50$.

Similar information for the case of a clamped-free column is given in Fig. 3. For these end conditions, the first-order and direct results differ less than $5 \%$ until $\eta$ values of 0.18 and 0.33 for a crack located at $\beta=0.25$ and $\beta=0.50$, respectively.

For a clamped-pinned column with a crack located at $\beta$ $=0.25$ [Fig. 4(a)], the differences between the buckling load obtained with the direct method and the perturbation technique is always less than $3 \%$. Note that in this case, the crack has a little effect on the buckling load, because of the bending moment transmitted by this section is very small (the bending moment is null at $\xi=0.30$ ). For a crack located at $\beta=0.50$, [Fig. 4(b)], the first-order solution differs from the direct solution less than $5 \%$ until values of crack severity of $\eta=0.25$.

Finally, the results corresponding to a clamped-clamped column are given in Fig. 5. When the crack section is located at $\beta=0.25$ [Fig. 5(a)], the crack has no effect on the first buckling load due to the null bending moment at the cracked section. This fact is recognized by the two solution methods considered. For a crack located at $\beta=0.50$, [Fig. 5(b)], the first-order solution differs from the direct solution less than $5 \%$ for values of crack severity less that $\eta=0.16$.
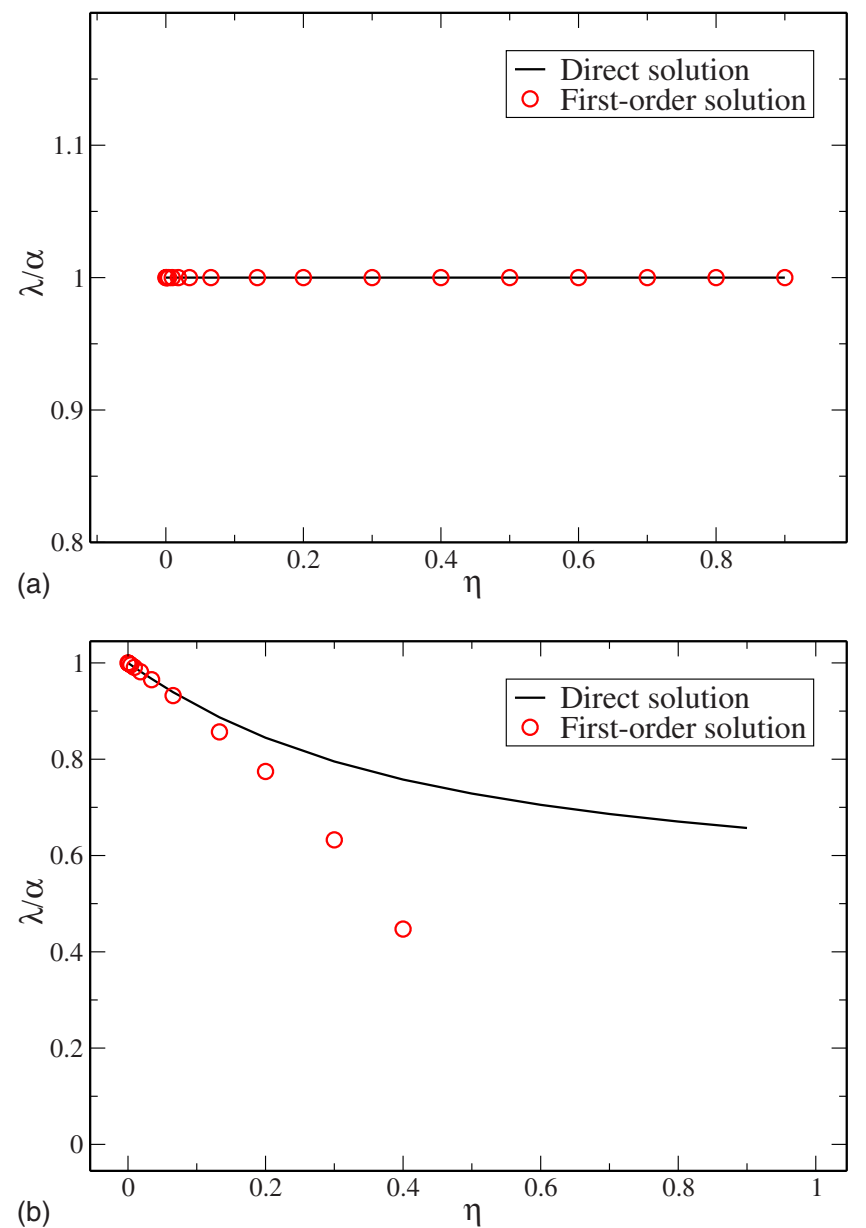

Fig. 5. First buckling load for different values of crack severity for a cracked clamped-clamped column: (a) crack located at $\beta=0.25$; (b) crack located at $\beta=0.50$

\section{Conclusions}

The perturbation method has been used in this work to reach closed-form solutions for the buckling loads of Euler-Bernoulli weakened columns with different boundary conditions. The model of cracked column used is based on the well-known method consisting of dividing the column into two segments connected by a rotational spring whose flexibility is related to the bending moment transmitted by the cracked section.

The first-order approximated solutions provided by the perturbation method have been compared with those found with the direct method for different boundary conditions, cracked-section position, and crack severity, showing in each case the validity limits of this approach.

\section{References}

Adams, R., Cawley, P., Pye, C., and Stone, B. (1978). "A vibration technique for non-destructive assessing the integrity of structures." Proc. Inst. Mech. Eng., Part C: J. Mech. Eng. Sci., 20, 93-100.

Biondi, B., and Caddemi, S. (2005). "Closed form solutions of EulerBernoulli beams with singularities." Int. J. Solids Struct., 42, 30273044.

Biondi, B., and Caddemi, S. (2007). "Euler-Bernoulli beams with multiple singularities in the flexural stiffness." Eur. J. Mech. A/Solids, 26, 789-809. 
Caddemi, S., and Caliò, I. (2008). "Exact solution of the multi-cracked Euler-Bernoulli column." Int. J. Solids Struct., 45, 1332-1351.

Fernández-Sáez, J., and Navarro, C. (2002). "Fundamental frequency of cracked beams: an analytical approach.” J. Sound Vib., 256, 17-31.

Fernández-Sáez, J., Rubio, L., and Navarro, C. (1999). “Approximate calculation of the fundamental frequency for bending vibrations of cracked beams." J. Sound Vib., 225, 345-352.

Freund, L. B., and Herrmann, G. (1976). "Dynamic fracture of a beam or plate in plane bending." Trans. ASME J. Appl. Mech., 43, 112-116.

Krawczuk, M., Palacz, M., and Ostachowicz, W. (2003). "The dynamic analysis of cracked Timoshenko beams by spectral element method." J. Sound Vib., 264, 1139-1153.

Loya, J., Rubio, L., and Fernández-Sáez, J. (2006). "Natural frequencies for bending vibrations of Timoshenko cracked beams." J. Sound Vib., 290, 640-653.

Morassi, A. (1993). "Crack-induced changes in eigenfrequencies of beam structures.” J. Eng. Mech., 119(9), 1798-1803.

Narkis, Y. (1994). "Identification of crack location in vibrating simply supported beams." J. Sound Vib., 172, 549-558.

Tada, H., Paris, P., and Irwin, G. (1985). The stress analysis of cracks handbook, 2nd Ed., Paris Productions, St. Louis.

Timoshenko, S., and Gere, J. (1965). Theory of elastic stability, McGrawHill, New York.

Wang, C. Y., Wang, C. M., and Aung, T. M. (2004). "Buckling of a weakened column." J. Eng. Mech., 130(11), 1373-1376. 